\title{
Dietary advice to cardiovascular patients. A brief update for physicians
}

\author{
Francesco Visioli ${ }^{1,2}$, Andrea Poli ${ }^{3}$ \\ ${ }^{1}$ Department of Molecular Medicine, University of Padua, Italy; ${ }^{2}$ IMDEA-Food, CEI UAM+CSIC, Madrid, Spain; \\ ${ }^{3}$ Nutrition Foundation of Italy, Milan, Italy
}

\begin{abstract}
It is important, in our opinion, to provide physicians with a brief update of scientifically-sound evidence in preventive nutrition, to be employed in their everyday practice, since the latest scientific and clinical advances in this area are generally not well known. Here, we review the most recent evidence in support of an optimal cardio-protective diet, and we identify the need to focus mainly on protective food which should be part of such diet, rather than on nutrients with negative effects to be limited (salt, saturated fats, simple sugars). We conclude that, to favor patient compliance, it is also necessary to underscore indications on the topics for which there is convincing and coherent literature, leaving other less-explored aspects to individual preferences.
\end{abstract}

\section{Introduction}

It is important to provide physicians with updated, i.e. based on current evidence clinical guidelines. Such guidelines could be then transposed into sound advice to the lay public. As far as pharmacology and therapeutics are concerned, the lay public is generally unaware of the latest scientific and clinical advances. This is quite

Correspondence: Andrea Poli, Nutrition Foundation of Italy, Viale Tunisia 38, 20124 Milano, Italy.

E-mail: poli@nutrition-foundation.it

Key words: Cardiovascular disease; low density lipoprotein; diet; fat; carbohydrates.

Acknowledgments: Supported in part by POR FESR 3S4H (to FV).

Conflict of interest: FV declares no conflict of interest associated with this publication. AP is President of NFI-Nutrition Foundation of Italy, a no profit Association partly supported by 18 large food companies.

Received for publication: 25 March 2019.

Accepted for publication: 5 April 2019.

CC Copyright F. Visioli and A. Poli, 2019

Licensee PAGEPress, Italy

Monaldi Archives for Chest Disease 2019; 89:1071

doi: 10.4081/monaldi.2019.1071

This article is distributed under the terms of the Creative Commons Attribution Noncommercial License (by-nc 4.0) which permits any noncommercial use, distribution, and reproduction in any medium, provided the original author(s) and source are credited. different from the nutrition area, in which the press and the Internet very often convey messages that can confuse patients [1]. A paradigmatic example is that of the role of total and, in particular, saturated fats whose role in human pathology is yet to be elucidated while contradictory messages abound. Something similar is happening for carbohydrates (complex vs simple) and their effects on blood glucose oscillations. Salt is also being re-appraised and new upper limits of consumption are being proposed. Clinicians, usually marginally interested in these issues, are often confused and find it difficult to provide correct advice to patients.

In this complex context, we think it important to provide physicians with a brief update of scientifically-sound evidence, to be employed in their everyday practice. To favor patient compliance, it is necessary to underscore indications on the topics for which there is convincing and coherent literature, leaving other less-explored aspects to individual preferences.

\section{Carbohydrates}

Carbohydrates (starches and sugars) should cover, according to most published guidelines, as much as $50-60 \%$ of the total daily caloric needs. Increasing evidence is actually focusing on the quality in addition to the quantity of these macronutrients.

Starches, which represent the prevalent share of carbohydrates that we introduce with food, are made of long chains of glucose molecules, which then enter into the bloodstream after digestion. This phenomenon may be characterized by a variable velocity and is influenced by various parameters typical of starch (its structure, the technological treatment used for the preparation, cooking times and conditions), but also by the presence or absence in the food or in the dish with which the starches are prepared, of fiber, fat, or other components able to influence the speed of gastric emptying.

Altogether, these processes create the "glycemic response" following the consumption of foods; this response depends on the glycemic index (GI) of foods and their quantity, in addition to the individual's genetics. A few years ago, an International Consensus Conference defined the favorable effects of a diet selectively enriched in foods with a low glycemic index (and therefore a reduced glycemic response) on cardiovascular and metabolic risk [2]. Neoplastic risk also appears to be favorably influenced by the preferential choice of items with a low glycemic index [3], probably due to the reduced insulin response induced by these foods (insulin, as we know, presents structural similarities with a family of growth factors, the IGFs, whose levels, according to some studies of observational epidemiology, correlate with a mild increase in neoplastic risk [4].

A recent paper underscores the possible protective effect of whole grains, as a source of carbohydrates, on CHD risk, which can be observed both in observational studies and in intervention 
trials [5]. The GI, in this study, appears to play a minor role, or no role at all.

It is also interesting to note that the carbohydrates content of the diet - contributing $>60 \%$ of energy - was associated with a poor prognosis (increased all-cause mortality and cardiovascular mortality) in the PURE study [6]. It is however likely that, in the Far East rural countries that contribute up to $2 / 3$ of the population enrolled in this study, a very high carbohydrate intake may be just a marker of a poor diet, still largely based on boiled rice, and that the excess mortality observed in these populations may actually reflect more the scarce presence of other nutrients (e.g. good quality proteins) rather than a true risk associated with a carbohydrate excess [7].

The role of sucrose in food (especially in soft drinks, where it is an example of the so-called "empty calories", i.e. without micronutrients or bioactive substances) is the subject of much attention in the nutritional world and in the media. For example, its contribution to the overweight appears to be less elucidated than what is suggested by most scientific literature and almost all of the lay press. A metaanalysis commissioned by the WHO concludes unambiguously that the consumption of sucrose in an isocaloric substitution of other nutrients (fats and proteins) is not associated with weight gain in intervention studies conducted according to a rigorous methodology, while addition of sucrose to a nutritional pattern does induce weight gain [8]. Recently, sucrose consumption has been associated with increased risk of coronary heart disease and modest increases in blood pressure $[8,9]$. The levels of consumption identified as critical in the first study (15\% or more of total calories) are quite high for countries where traditional diets are still adhered to [10]. No association between dietary sucrose, calculated using on objective urinary marker of daily intake, and CHD incidence or mortality has - on the other hand - been observed in a recent reanalysis of the Women Health Initiative (WHI) study data [10].

The heated debate on these issues led the WHO to abandon the proposal to recommend a reduction of $5 \%$ of daily calories from added sugars (mostly sucrose), because of the scant evidence in support this proposal.

\section{Dietary fat}

Data from the literature, starting from the results of the aforementioned large randomized intervention trial WHI [11] have shown that limiting the intake of total fat does not significantly improve the cardiovascular risk profile, the incidence of type 2 diabetes and, likely, does not induce significant weight loss. Consequently, no significant protective effect on cardiovascular end-points was observed, in this trial, among women who cut their total fat intake from $35 / 37 \%$ to $25 / 27 \%$ for an average of 7 years, as compared to women who did not change their diet [11].

The focus must, therefore, shift to the adequate consumption of individual categories of edible fats. Even in this context the most recent evidence has suggested some changes in paradigms (widely taken up by the non-specialized press) that deserve to be discussed.

The dietary intake of saturated fatty acids - long considered as an important determinant of LDL cholesterolemia and, therefore, of cardiovascular risk - must indeed be limited, without however creating nutritional imbalances and without leading to the ban of any kind of otherwise healthful food [12]. Two meta-analyses, in fact, reported no association between saturated fat intake and overall mortality [12]. A pertinent example is that of milk and derivatives (typically rich in saturated fatty acids), whose consumption, in light of the data available to date, should not be discouraged [13]. Milk (probably due to its content of calcium and/or tripeptides with a mild ACEinhibitory action), might, in fact, induce a reduction of blood pressure and, therefore, of the risk of cerebrovascular events, without significantly affecting the risk of coronary events $[13,14]$. Those who eliminate milk from their diet (generally due to real or presumed intolerance) might experience a significant increase in the risk of developing diabetes or hypertension (one new case for every 15 subjects who stop using milk) [15], thus theoretically exposing themselves to the vascular complications of these conditions. Cheese intake, in a number of observational studies, is associated with a reduction of CHD events and stroke [13]. In the context of a varied and balanced diet, the consumption of milk (and probably of dairy products) does not, therefore, significantly influence cardiovascular risk and should not be discouraged [13].

On the other hand, the ban on the consumption of trans unsaturated fatty acids, typical of old margarines used in dough and some low-quality baked goods should be implemented. The consumption of trans fatty acids (generally identified on the label as "partially hydrogenated vegetable fats") leads to untoward effects on lipid and lipoprotein metabolism, i.e. increase in LDL-related cholesterol, reduction of HDL-related cholesterol, deterioration of endothelial function, perhaps due to the pro-inflammatory action they induce; the direct association between their food consumption and coronary risk is also well documented [16]. A paradigmatic example is that of Countries where intake of trans fatty acids - and cardiovascular events - are decreasing following an ad-hoc ban [17].

Consumption of monounsaturated fatty acids (typically found in olive oil, but also in foods of animal origin such as chicken and pork meat) does not seem to exert particular effects in cardiovascular prevention, contrary to popular wisdom. The effect of monounsaturates on total and LDL cholesterolemia is very modest and their correlation with coronary risk, at best, uncertain $[17,18]$. A sub-analysis of the PREDIMED study suggests that increasing consumption non-virgin or extra virgin olive oil, is associated with a significant reduction in coronary risk and mortality for all causes, while increasing consumption of common (non-virgin or non-extra virgin olive oil was associated with a non-significant increase of both these parameters [19]. The available evidence thus confirms the protective effect of the consumption of extra virgin olive oil in relation to coronary risk, but tends to attribute this protective effect to its (poly)phenols and not to its lipid components such as oleic acid [18]. Oleic acid is also non-essential and its consumption is on average already quite high. A new line of research is trying to discriminate the effects of plant-derived monounsaturates from those of the meat-derived ones [20]. The robustness of this approach is, however, in need of confirmation.

As opposed to monounsaturates, polyunsaturated fatty acids are essential in that the human organism is not able to synthesize omega- 3 and omega- 6 . Their intake with the diet is, hence, very important. An adequate dietary intake of omega- 3 from plant foods (linseed, canola, and soybean oils and walnuts) namely alpha linolenic acid (ALA) and from fish eicosapentaenoic and docosahexaenoic acids (EPA and DHA, respectively) is associated with a significant reduction of coronary risk and sudden death [21], especially in the elderly [22]. These preventive effects, associated in most observational studies to fish consumption, are classically explained by favorable effects of these fatty acids on triglyceridemia, on platelet function, on blood pressure, and on the lower production of adhesion and proinflammatory proteins by the arterial wall, in addition to direct antiarrhythmic and antioxidant effects [23]. An Italian study (the GISSI-Prevenzione), in which capsules of fish oil (at a dose of $850 \mathrm{mg} /$ day) or a placebo were administered to subjects with a personal clinical history of a coro- 
nary event, was the first one to clinically confirm the cardioprotective effects of EPA and DHA [24]. After this study, no further and significant evidence emerged, from controlled trials, in favor of treatment with omega-3 in secondary prevention. It is important to underline that these negative outcomes pertain to supplementary fish oil, not to the advice to regularly consume fish or plant sources of ALA. Several reasons may, in fact explain the lack of effect reported by the most recent trials: i) cardiopathic patients currently receive highly effective multi-drug pharmacological treatments; the effect of adding "on top" of these treatments omega- 3 fatty acids is likely very small; ii) patients who could benefit more than others from a treatment with omega-3 (for example those with low basal blood levels of these fatty acids) have never been identified and selected, with the risk of "not seeing" the effects of the supplementation [25]. From a dietary point of view, bi-weekly consumption of fish (especially fatty fish) remains a cornerstone of cardiovascular prevention.

The dietary intake of omega- 6 fatty acids, e.g. the substitution of $5 \%$ of calories from saturates with linoleic acid was associated with a significant reduction in cardiovascular risk $(-9 \%)$ in a US metaanalysis [26]. Also, plasma levels of linoleic acid correlate negatively with cardiovascular risk and positively with insulin sensitivity [26]. High plasma concentrations of linoleic acid are also associated with a $\sim 50 \%$ reduction of the risk of developing diabetic disease in the following five years. The abundant presence of these fatty acids in dried fruit and nuts can also contribute to explain the protective effect found in the "nuts" arm of the PREDIMED study [27].

Alas, the idea that omega- 6 fatty acids are pro-inflammatory is still widespread, especially in the nutritional world. A systematic review of the literature did not actually detect any increase in inflammatory markers associated with the consumption of omega6 in humans [28]. The idea that their competitive relationship with omega-3s should be kept as low as possible is also commonplace, but it is merely based on biochemical considerations (omega- 6 compete with omega-3 for some enzymatic activities - elongase and desaturase - and are the precursors of leukotrienes and thromboxanes) [29]. Of note, the majority of human studies do not confirm this biochemical hypothesis. In a case-control study of Italian infarcted subjects, high plasma levels of both omega-3 and omega- 6 were associated with a clear risk reduction, while the omega- 6 omega- 3 ratio did not correlate significantly with the risk itself [30].

The intake of omega- 6 fatty acids is, moreover, quite low in Italy and Europe. According to an INRAN-SCAI survey, in which omega-6s were unfortunately reported together with omega-3 polyunsaturates, the cumulative omega- 6 and omega- 3 intake covers only $4-5 \%$ of total calories [30]. An increase in their dietary intake, from seed oils, vegetables and nuts, could help to further reduce coronary risk in countries where consumption is sub-optimal.

In summary, the cardio-protective diet calls for a reduction (but not elimination) of the share of saturated fats and its replacement with polyunsaturated omega-3 (from fish) and omega-6 (from seeds, oils, vegetables, whole grains, and poultry). The intake of unsaturated fatty acids with a trans-conformation of industrial origin (generally already low in Italy) should be minimized.

\section{Alcohol}

The effects of alcohol, i.e. ethanol, on health are variegated. While an excessive consumption of alcoholic beverages has serious untoward effects, both physically and socially, there is now well-established epidemiological evidence, accompanied by biochemical and mechanistic data, that correlate moderate alcohol consumption with favorable effects on the risk of some common diseases, mostly atherosclerosis [31]. We emphasize that both the evidence relating to the beneficial effects of moderate alcohol consumption and those relating to unfavorable effects on some pathologies (tumors) derive from epidemiological (cohort or casecontrol) studies, which do not allow to establish causal links. Residual confounding factors might remain in the analyses [31].

Keeping this in mind, we can confidently propose that moderate consumption of alcohol over time ( $\leq 2-3$ drinks/day for men and $\leq 1-2$ drinks/day for women and the elderly, remembering that - by international agreement - a drink corresponds to a standard glass of $150 \mathrm{~mL}$ wine, or to a can of $330 \mathrm{~mL}$ beer, or to a standard dose of $40 \mathrm{~mL}$ of liquor) is associated with lower morbidity and mortality due to cardiovascular ischemic events. Adverse consequences on other pathologies (in particular on neoplastic mortality) exist, but they are quantitatively small within these levels. The advantageous effects on cardiovascular events translates into a reduction of allcause mortality for any cause, with a nadir seen for consumption levels of 1-2 drinks/day [31]. In women, slight but significant increases in breast cancer incidence can be seen also at these low intake levels: but according to recent data, they are observed only in women with a family breast cancer history, or in women reporting binge drinking episodes.

The risk of developing diabetic disease and, maybe, some types of dementia also appears to be favorably influenced at such consumption levels. We also would like to remind readers that - in terms of effect - there are no major differences in effect between wine (white or red), beer and liqueurs: the effects are very likely due to alcohol and not to the minor components of the different drinks [32].

Even though the evidence is in favor of moderate consumption of alcohol, patients who do not drink should not be encouraged to start to do so for health reasons; those who drink in moderate quantities and are healthy can, instead, be reassured [31].

\section{Salt}

Restriction of salt intake, which in developed countries often exceeds 10 grams/day, is one of the cornerstones of cardio-and especially cerebro-vascular risk reduction. Proponents of such guideline explain it by blood pressure reduction. The "active" component of salt is sodium, which directly depresses endothelial function (an effect that can be partially neutralized by a high intake of potassium, according to recent studies), and might carry out proinflammatory actions.

However, some recent studies are suggesting that the correlation between sodium and cardiovascular risk follows a "J" or "U" curve: low or very low consumption levels are also associated with risk [33]. Although it is possible to explain this apparent contradiction with "reverse causality" (subjects with the lowest sodium intake are often unhealthy and restrict their sodium intake following a recommendation by their physician: the cause of the observed excess death rate in these patients would hence not be their low salt intake, but rather the presence of clinical conditions which led the treating physician to suggest salt restriction), the emphasis on a more pronounced sodium restriction is being attenuated worldwide. In the USA, for example, the Institute of Medicine suggests 2.3 grams/day (corresponding to about 6 grams/day of salt) as a desirable intake level. Conversely, a Cochrane Review [34] stresses that even lower 
levels of intake are unlikely to provide any benefit. The debate on this issue is still open [35].

\section{Dietary or supplementary fiber}

There is a general consensus that an optimal diet should include a large proportion of fiber-rich foods. In particular, we should eat polysaccharides deriving from plant cells resistant to digestion by human enzymes that, therefore, reaches the small intestine and the colon unchanged.

The term "fiber" actually encompasses a heterogeneous set of compounds, with varied characteristics and, hence, functional properties. Some fiber forms very viscous gels when in an aqueous environment, in turn slowing down stomach emptying, delaying absorption of some nutrients in the small intestine (mostly simple sugars and triglycerides, especially in the post-prandial phase) and lowering, to different extents, cholesterol concentrations [36]. Viscous fibers include pectin, beta-glucans, some gums e.g. guar gum, and mucilage e.g. psyllium. Most fibers are also rapidly metabolized, i.e. fermented by the microbiota, bacteria that reside in the small intestine and in the colon. In addition to selectively increasing the amount and diversity of colonic bacteria, favoring the development of the strains that use them as a preferential energy source (a "prebiotic" effect) fiber fermentation by the microbiota leads to the formation of short chain fatty acids (SCFA) such acetate, propionate, or butyrate and carbon dioxide. SCFA can be absorbed and metabolized to produce energy (in a way revisiting the old notion that vegetable fiber does not provide calories): butyrate is very important form mucosal trophism and appears to be the preferred energy source for epithelial cells lining the colon [37,38]. Pectins, beta-glucans, guar gum, inulin, and oligofructose are rapidly fermented, while cellulose and lignin are more resistant to fermentation. Foods rich in fermentable fiber are oats and barley, as well as fruit and vegetables. Cereal fiber rich in cellulose, such as wheat bran, is instead relatively resistant to bacterial fermentation.

Subjects with high $(-33 \%$ for consumption increases of 10 $\mathrm{g}$ /day) fiber intake witness a reduced coronary risk, mostly yet not exclusively consequent due to the beneficial effect of fiber on cholesterolemia [36]. The European Food Safety Authority (EFSA) has indeed granted a specific claim to beta-glucan, to be used at a dose of 3 grams/day.

Physicians should also be aware of interactions between fibers and drugs. Examples include psyllium, which can reduce the absorption of digoxin and warfarin; guar gum, which can slow the absorption of digoxin and bumetanide; and pectin, which can reduce the absorption of lovastatin when the two are taken at the same time. In general, medications should be taken at least an hour before or two hours after fiber supplements use.

\section{Conclusions}

We wanted to provide physicians such as clinical cardiologists with some updated evidence, that they might want to incorporate into their practice. The first one is not to reduce the overall intake of dietary fat (except in cases of excessive consumption); eliminate from the diet trans unsaturated fats (spelt as "partially hydrogenated vegetable fats" on the label) and reduce, to some extent, animal, saturated fats. Replace the eliminated fats with extra virgin olive oil or with oils or foods rich in polyunsaturated (both omega-6, like most seed oils, and omega-3, of which the fat fish is rich) fatty acids. It is also time to de-emphasize attention on foods rich in cholesterol (eggs, shellfish); correlations between the consumption of dietary cholesterol, cholesterolemia, and cardiovascular risk are, in fact, very weak [39].

Maintain an adequate, isocaloric intake of starch, namely pasta, rice, and (in lower amounts) bread or other starchy foods. Whole grains should be preferred over refined ones. Foods with a lower glycemic response (low glycemic index in quantities that translate into low glycemic load) should be preferred to those with greater response. The consumption of sugar and sweet foods or sugar-sweetened beverages should be limited with no phobias. There is no reason to discourage moderate alcohol consumption, i.e. $\leq 2-3$ drinks/day for males and $\leq 1-2$ for females, if there is no risk of abuse. Even if not discussed in this paper and in the frame of a calorie-appropriate diet, the use of small amounts of chocolate, dried fruit, berries, coffee or tea in proper amounts should be encouraged because it provides healthful (poly)phenols [40,41]. The appropriate use of supplements should also be envisioned because it is highly appealing to patients [1].

Finally, physicians should focus on the whole diet and lifestyle rather than individual food items and their bioactive components. Empathy and keen attention to individuals' behavior likely amplify the effects of healthful diets.

\section{References}

1. Khedkar S, Carraresi L, Bröring, S. Food or pharmaceuticals? Consumers' perception of health-related borderline products. PharmaNutrition 2017;5:133-40.

2. Augustin LS, Kendall CW, Jenkins DJ, et al. Glycemic index, glycemic load and glycemic response: An International Scientific Consensus Summit from the International Carbohydrate Quality Consortium (ICQC). Nutr Metab Cardiovasc Dis 2015;25:795-815.

3. $\mathrm{Hu} \mathrm{J}$, La Vecchia C, Augustin LS, et al. Glycemic index, glycemic load and cancer risk. Ann Oncol 2013;24:245-51.

4. Salvioli S, Capri M, Bucci L, et al. Why do centenarians escape or postpone cancer? The role of IGF-1, inflammation and p53. Cancer Immunol Immunother 2009;58:1909-17.

5. Reynolds A, Mann J, Cummings J, et al. Carbohydrate quality and human health: a series of systematic reviews and metaanalyses. Lancet 2019;393:434-45.

6. Dehghan M, Mente A, Zhang X, et al. Associations of fats and carbohydrate intake with cardiovascular disease and mortality in 18 countries from five continents (PURE): a prospective cohort study. Lancet 2017;390:2050-62.

7. Hansel B, Roussel R, Giral P. Associations of fats and carbohydrates with cardiovascular disease and mortality-PURE and simple? Lancet 2018;391:1680.

8. Te Morenga L, Mallard S, Mann J. Dietary sugars and body weight: systematic review and meta-analyses of randomised controlled trials and cohort studies. Brit Med J 2012;346:e7492.

9. Yang Q, Zhang Z, Gregg EW, et al. Added sugar intake and cardiovascular diseases mortality among US adults. JAMA Intern Med 2014;174:516-24.

10. Sette S, Le Donne C, Piccinelli R, et al. The third Italian National Food Consumption Survey, INRAN-SCAI 200506-part 1: nutrient intakes in Italy. Nutr Metab Cardiovasc Dis 2011;21:922-32.

11. Howard BV, Van Horn L, Hsia J, et al. Low-fat dietary pattern 
and risk of cardiovascular disease: the Women's Health Initiative Randomized Controlled Dietary Modification Trial. JAMA 2006;295:655-66.

12. Siri-Tarino PW, Sun Q, Hu FB, Krauss RM. Meta-analysis of prospective cohort studies evaluating the association of saturated fat with cardiovascular disease. Am J Clin Nut. 2010;91:535-46.

13. Visioli F, Strata A. Milk, dairy products, and their functional effects in humans: a narrative review of recent evidence. Adv Nutr 2014;5:131-43.

14. Soedamah-Muthu SS, Ding EL, Al-Delaimy WK, et al. Milk and dairy consumption and incidence of cardiovascular diseases and all-cause mortality: dose-response meta-analysis of prospective cohort studies. Am J Clin Nutr 2011;93:158-71.

15. Nicklas TA, Qu H, Hughes SO, et al. Self-perceived lactose intolerance results in lower intakes of calcium and dairy foods and is associated with hypertension and diabetes in adults. Am J Clin Nutr 2011;94:191-8.

16. Mozaffarian D, Aro A, Willett WC. Health effects of transfatty acids: experimental and observational evidence. Eur J Clin Nutr 2009;63:S5-21.

17. Wright M, McKelvey W, Curtis CJ, T et al. Impact of a municipal policy restricting trans fatty acid use in New York City restaurants on serum trans fatty acid levels in adults. Am J Public Health 2019;109:634-6.

18. Visioli F, Franco M, Toledo E, et al. Olive oil and prevention of chronic diseases: Summary of an International conference. Nutr Metab Cardiovasc Dis 2018;28:649-56.

19. Guasch-Ferre M, Hu FB, Martinez-Gonzalez MA, et al. Olive oil intake and risk of cardiovascular disease and mortality in the PREDIMED Study. BMC Med 2014;12:78.

20. Guasch-Ferre M, Zong G, Willett WC, et al. Associations of monounsaturated fatty acids from plant and animal sources with total and cause-specific mortality in two US prospective cohort studies. Circ Res 2019 doi: 10.1161/CIRCRESAHA.118.313996. [Epub ahead of print].

21. Mozaffarian D, Rimm EB. Fish intake, contaminants, and human health: evaluating the risks and the benefits. JAMA 2006;296:1885-99.

22. Visioli F, Hagen TM. Nutritional strategies for healthy cardiovascular aging: focus on micronutrients. Pharmacol Res 2007;55:199-206.

23. Giordano E, Visioli F. Long-chain omega 3 fatty acids: molecular bases of potential antioxidant actions. Prostaglandins Leukot Essent Fatty Acids 2014;90:1-4.

24. Dietary supplementation with n-3 polyunsaturated fatty acids and vitamin E after myocardial infarction: results of the GISSIPrevenzione trial. Gruppo Italiano per lo Studio della Sopravvivenza nell'Infarto miocardico. Lancet 1999;354:447-55. 25. von Schacky C. Omega-3 fatty acids in cardiovascular dis- ease - an uphill battle. Prostaglandins Leukot Essent Fatty Acids 2015;92:41-7.

26. Farvid MS, Ding M, Pan A, et al. Dietary linoleic acid and risk of coronary heart disease: a systematic review and meta-analysis of prospective cohort studies. Circulation 2014;130:1568-78.

27. Estruch R, Ros E, Salas-Salvado J, et al. Primary prevention of cardiovascular disease with a mediterranean diet supplemented with extra-virgin olive oil or nuts. N Engl J Med 2018;378:e34.

28. Johnson GH, Fritsche K. Effect of dietary linoleic acid on markers of inflammation in healthy persons: a systematic review of randomized controlled trials. J Acad Nutr Diet 2012;112:1029-41, 1041 e1-15.

29. Poli A, Visioli, F. Recent evidence on omega 6 fatty acids 11 and cardiovascular risk. Eur J Lipid Sci Techn 2015;117:1847-52.

30. Marangoni F, Novo G, Perna G, et al. Omega-6 and omega-3 polyunsaturated fatty acid levels are reduced in whole blood of Italian patients with a recent myocardial infarction: the AGEIM study. Atherosclerosis 2014;232:334-8.

31. Poli A, Marangoni F, Avogaro A, et al. Moderate alcohol use and health: a consensus document. Nutr Metab Cardiovasc Di. 2013;23:487-504.

32. Visioli F. The resveratrol fiasco. Pharmacol Res. 2014;90:87.

33. O'Donnell M, Mann JF, Schutte AE, et al. Dietary sodium and cardiovascular disease risk. N Engl J Med 2016;375:2404-6.

34. Adler AJ, Taylor F, Martin N, et al. Reduced dietary salt for the prevention of cardiovascular disease. Cochrane Database Syst Rev 2014:CD009217.

35. Lelli D, Antonelli-Incalzi R, Bandinelli S, et al. Association between sodium excretion and cardiovascular disease and mortality in the elderly: A Cohort Study. J Am Med Dir Assoc 2018;19:229-34.

36. Liu L, Wang S, Liu J. Fiber consumption and all-cause, cardiovascular, and cancer mortalities: a systematic review and metaanalysis of cohort studies. Mol Nutr Food Res 2015;59:139-46.

37. Wong JM, de Souza R, Kendall CW, et al. Colonic health: fermentation and short chain fatty acids. J Clin Gastroenterol 2006;40:235-43

38. Flach J, Koks M, van der Waal MB, et al. Economic potential of probiotic supplementation in institutionalized elderly with chronic constipation. PharmaNutrition 2018;6:198-206.

39. Abbasi J. New cholesterol guidelines personalize risk and add treatments. JAMA 2019 doi: 10.1001/jama.2018.21019. [Epub ahead of print].

40. Visioli F, Davalos A. Polyphenols and cardiovascular disease: a critical summary of the evidence. Mini Rev Med Chem 2011;11:1186-90.

41. Tome-Carneiro J, Visioli F. Polyphenol-based nutraceuticals for the prevention and treatment of cardiovascular disease: Review of human evidence. Phytomedicine 2016;23:1145-74. 